\title{
Droplets Propel on Hot Oil
}

\author{
When placed on hot oil films, water droplets self-propel as they boil off, \\ reaching speeds significantly faster than those achieved via most other \\ self-propulsion mechanisms.
}

\section{By Erika K. Carlson}

S elf-propelling droplets could be used to develop microrobots or self-cleaning surfaces. So far, however, their use is limited, as most of the discovered droplet-propulsion methods achieve speeds of only a few millimeters per second. Now, Victor Julio Leon and Kripa Varanasi of the Massachusetts Institute of Technology have demonstrated a method to move droplets roughly a hundred times faster, propelling droplets at speeds on the order of 10 $\mathrm{cm} / \mathrm{s}$ [1]. This speedup could be beneficial for cleaning, for example, because researchers are interested in developing ways to quickly remove contaminants, such as salts, from a surface to avoid substance buildup and damage.

In their experiments, Leon and Varanasi dropped water droplets a couple of millimeters wide onto heated solid surfaces covered in thin oil films. They then captured the droplets' motions with a high-speed camera and analyzed the footage. The duo observed that following deposition, the droplets immediately started moving in a random direction.

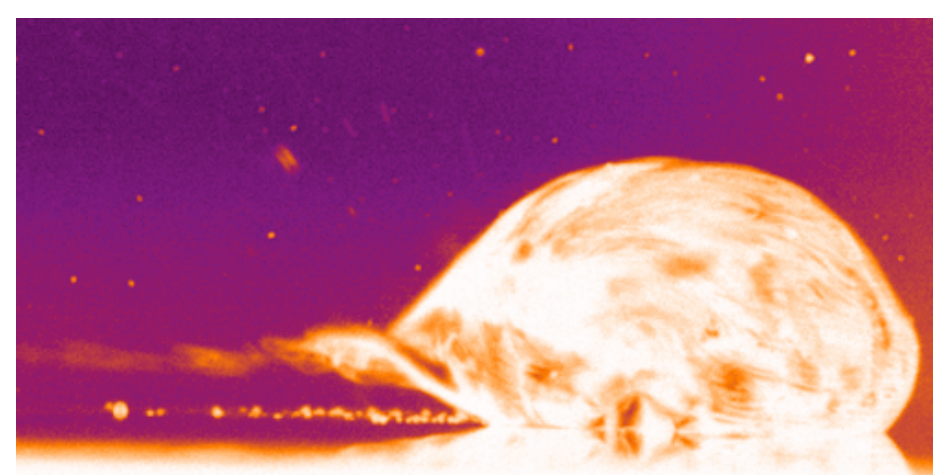

Credit: V. J. Leon
Video 1: A water droplet placed on a surface coated with a hot oil film can self-propel at speeds on the order of $10 \mathrm{~cm} / \mathrm{s}$.

Credit: V. J. Leon and K. K. Varanasi [1]

From their footage, Leon and Varanasi observed that many factors, including oil temperature and droplet viscosity, affected the droplets' speeds. They also determined the propulsion mechanism, which they say is ejection of water vapor from one side of the droplet. They found that when each water droplet hit the hot oil, vapor bubbles started forming at one point on the droplet-oil interface and then exited the droplet.

Subsequent bubbles tended to form near those first ones, such that a single vapor trail formed behind the droplet, pushing it in a preferred direction. Another observation they made was that the droplets stayed in contact with the oil surface, which they say helped the vapor eject in one direction and contributed to the droplets' fast speeds.

Erika K. Carlson is a Corresponding Editor for Physics based in New York City.

\section{REFERENCES}

1. V. J. Leon and K. K. Varanasi, "Self-propulsion of boiling droplets on thin heated oil films," Phys. Rev. Lett. 127, 074502 (2021). 